TITLE:

\title{
Three-dimensional observation of an helical hot structure during a sawtooth crash in the WT-3 tokamak
}

\section{$\operatorname{AUTHOR}(\mathrm{S})$ :}

Yamaguchi, S; Igami, H; Tanaka, H; Maekawa, T

\section{CITATION:}

Yamaguchi, S ...[et al]. Three-dimensional observation of an helical hot structure during a sawtooth crash in the WT-3 tokamak. PHYSICAL REVIEW LETTERS 2004, 93(4): 045005.

\section{ISSUE DATE:}

2004-07-23

URL:

http://hdl.handle.net/2433/49901

\section{RIGHT:}

Copyright 2004 American Physical Society 


\title{
Three-Dimensional Observation of an Helical Hot Structure during a Sawtooth Crash in the WT-3 Tokamak
}

\author{
S. Yamaguchi, ${ }^{1}$ H. Igami, ${ }^{2}$ H. Tanaka, ${ }^{2}$ and T. Maekawa ${ }^{2}$ \\ ${ }^{1}$ Graduate School of Science, Kyoto University, Kyoto 606-8502, Japan \\ ${ }^{2}$ Graduate School of Energy Science, Kyoto University, Kyoto 606-8502, Japan \\ (Received 24 January 2004; revised manuscript received 30 March 2004; published 23 July 2004)

\begin{abstract}
Sawtooth crashes in an Ohmically heated plasma in the WT-3 tokamak have been observed by using soft x-ray computer tomography at three different poloidal cross sections around the torus. Initially, collapsing proceeds slowly with keeping the helical structure of an $m=1 / n=1$ hot core around the torus. It accelerates as the helical hot structure is strongly deformed and fades away in the manner that the hot core at the high field side becomes obscure and disappears, while that at the low field side is deformed into a thin crescent aligned along the inversion circle, which survives even at the completion of the crash.
\end{abstract}

The sawtooth oscillation is a regular periodic reorganization of the core plasma surrounding the magnetic axis in tokamaks as first observed by von Goeler et al. on the soft $\mathrm{x}$-ray signals in the symmetric tokamak [1]. Since then, it has been observed on many diagnostics in almost all tokamaks, large and small, under various different operation conditions corresponding to a broad range of plasma parameters. Thus, the sawtooth oscillation is a universal phenomenon in tokamak plasmas and naturally supposed to affect significantly the fusion burning [2]. It has been attracting strong interest and provoking continuous effort in both experimental and theoretical investigations to clarify the mechanism [3].

Kadomtsev first proposed a standard model for the sawtooth crash based on the magnetic reconnection induced by the shift of hot core onto the $q=1$ surface due to the $m=1 / n=1$ internal kink instability [4], where $m$ and $n$ are the poloidal and toroidal mode numbers, respectively. The model predicts that the hot plasma inside the $q=1$ surface is mixed up with the outside cooler plasma in the course of successive reconnection of the helical magnetic fluxes inside and outside the $q=1$ surface. After the reconnection is completed, the safety factor rises as $q(r)>1$ everywhere with $q(0)=1$ on the magnetic axis. Many experimental observations were, however, not quite consistent with the model [3]. Especially, a number of measurements on the $q(r)$ profile during sawtooth oscillations have shown that $q(0)$ does not rise above unity even just after the crash, indicating that complete reconnection does not take place [5-7], although some measurements have shown different results [8]. Along with these results, several crash models have been proposed and examined. A popular model is a modification of the single magnetic reconnection process to the process with two steps; initially, an internal kink mode causes some magnetic reconnection in the vicinity of the $q=1$ surface and then a secondary event responsible for the rapid collapse of the hot core takes place.
Pressure driven instability induced by the shift of hot core [9] or stochastization of the magnetic field structure triggered at a critical amplitude of the kink perturbation [10] were suggested and analyzed as a mechanism for the secondary event. Another model is the crash due to the local magnetic reconnection at the low field side [11].

A notable characteristic of these models may be the appearance of helical asymmetry during crash processes. An initial $m=1 / n=1$ kink mode generates an helical hot structure along the $q=1$ pitch line around the torus. As the crash process proceeds further, the developed helical structure induces the secondary events, which would be inherently much stronger at the bad curvature region of the low field side and an helical asymmetry would arise. These considerations and conjecture encouraged the authors to develop a multitoroidally positioned (MTP) soft $\mathrm{x}$-ray computerized tomography (SXCT) system from the single SXCT system in the WT-3 tokamak $[12,13]$ to realize the three-dimensional observation of sawtooth crashes. Such a three-dimensional observation is the first attempt and expected to bring out new information on the crash processes. In this Letter, we present the result, which visualizes a picture of whole helical hot structure around the torus during the crash.

The WT-3 tokamak (major and minor radii of $R_{0}=$ $65 \mathrm{~cm}$ and $a=20 \mathrm{~cm}$, respectively) has 16 port sections [Fig. 1(a)]. Three sets of the same SXCT system are fabricated at the $3 \mathrm{rd}, 8 \mathrm{th}$, and 15 th port sections around the torus, composing the MTP SXCT system. Each SXCT system consists of five soft X-ray (SX) cameras [Fig. 1(b)]. Each camera views the plasma with 20 chords with a slit and an array SX detector of 20 channels, which is sensitive to photon energies from 0.2 to $10 \mathrm{keV}$. The spatial structure of the SX emissivity (SX image) is reconstructed by computerized tomography (CT) based on the Fourier-Bessel expansion technique [14], which is suitable to the present circular plasma cross section in WT-3. In addition to the MTP SXCT system, a filter bank 
(a)

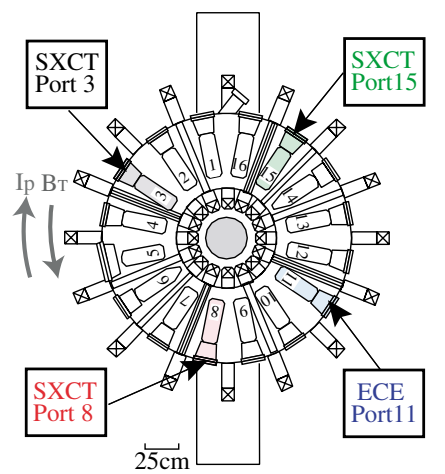

(b)

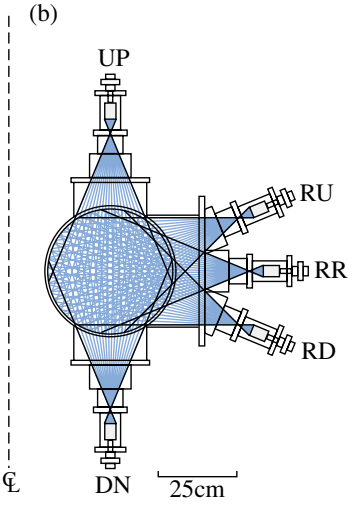

FIG. 1 (color online). (a) Top view of the WT-3 tokamak. (b) Cross-sectional view of the SXCT system.

radiometer is fabricated at the 11th radial port for the second harmonic electron cyclotron emission (ECE) measurement as shown in Fig. 1(a), providing supplementary information.

We have observed sawtooth crashes in the Ohmic discharges with a low safety factor of $q_{a} \simeq 2.7$ with plasma currents of $I_{p} \simeq 150 \mathrm{kA}$ and toroidal field of $B_{T}(0)=$ $1.4 \mathrm{~T}$ at $R=R_{0}$. The line averaged electron density and the central electron temperature are $\bar{n}_{e}=1.2 \times 10^{13} \mathrm{~cm}^{-3}$ and $T_{e}(0) \simeq 400 \mathrm{eV}$, respectively. A variety of crashes have appeared. Figure 2 shows the time evolutions of SX images for a typical case of the most frequently occurring types of crash. We notice that in the all port sections the hot core evolves into an $m=1$ mode structure and rotates in the clockwise direction, that is, in the electron diamagnetic direction, and finally fades away. The detailed evolutions are, however, quite different depending on the SXCT locations.

Better interpretation concerning the evolutions of SX images is obtained if we suppose the helical structure of an $m=1 / n=1$ hot core rotating toroidally around the torus as shown in Fig. 3 when we examine the SX images in Fig. 2 as follows. First $(t=63.595 \mathrm{~ms})$, a notable shift of the hot core to the low field side is seen on the SX image at port 15, while no appreciable shifts appear at ports 3 and 8. At the next time of $t=63.620 \mathrm{~ms}$, appreciable shifts appear in ports 8 and 15 and the shift to the low field side is remarkably enhanced as appeared at port 3 . These locations of hot cores on the SX images at this time are consistent with the helical hot structure of an $m=$ $1 / n=1$ kink mode as shown in Fig. 3, where spatial relationships between the $m=1 / n=1$ kink structure of hot core and the measuring ports of SXCT and ECE are schematically shown for various times in Fig. 2. The arrows on the hot cores in the SX images show the shift directions of hot cores of the $m=1 / n=1 \mathrm{kink}$ mode in the approximation of a straight plasma cylinder for the toroidal plasmas. At $t=63.650 \mathrm{~ms}$, the hot core at the low field side appears at port 8 , while the hot core at the high field side is at port 15 and the hot core at the lower side is at port 3, indicating that the helical hot

structure is rotating to the reverse direction of the plasma current as shown in Fig. 3. This rotation of the helical hot structure is also detected on the ECE signals as shown in Fig. 4. Namely, the ECE signal from the high (low) field side on the midplane at port 11 passes through a peak (bottom) at (2) $t=63.620 \mathrm{~ms}$ and a bottom (peak) at (7) $t=63.670 \mathrm{~ms}$, consistent with the corresponding locations of the helical hot structure around the torus.

Thus, the picture of toroidally rotating helical hot structure appears valid, at least during $t=63.620-$ $63.680 \mathrm{~ms}$, but hot cores themselves evolve quite differently depending on the shift directions in the poloidal cross section. At the beginning of the crash, the hot core at the low field side is pushed strongly to the inversion circle (port 3 at $t=63.620 \mathrm{~ms}$ ) and then the shape is deformed into a bean shape (port 8 at $t=63.650 \mathrm{~ms}$ ). Around this time the hot core at the high field side becomes obscure (port 15), suggesting that the helical hot structure begins to fade away. Finally, at the end of the crash $(t=63.690-63.710 \mathrm{~ms})$, there only exists a thin hot structure of crescent shape at the low field side (port 15), which is expanded along the inversion circle from the bottom to top sides through the low field side. No hot structures are seen on the SX images at ports 3 and 8. There is also an asymmetry between the hot cores at the upper and lower sides of the cross sections. Namely, the hot core at the upper side (port 8 at $t=63.630 \mathrm{~ms}$ ) becomes obscure at a relatively early stage of $t=$ $63.670 \mathrm{~ms}$ as observed at port 15 , while that at the lower side survives until $t=63.680 \mathrm{~ms}$ as observed at port 8 .

Figure 4(a) shows the time evolutions of total SX emissions integrated inside the inversion radius. They begin to decrease when the kink mode appears [(1) $t=$ $63.595 \mathrm{~ms}$ ] and decrease very slowly until (4) $t=$ $63.640 \mathrm{~ms}$, during which the helical hot structure is clearly seen. Collapsing accelerates as the hot core at the high field side begins to obscure [see port 15 at (5) $t=63.650 \mathrm{~ms}]$. In other words, the crash proceeds gradually in the first phase where the helical structure of the $m=1 / n=1$ hot core is maintained with the gradual increase of the shifts of hot cores, while it proceeds rapidly in accordance with the strong deformation and disappearance of the helical hot structure in the second phase of the crash.

Thus, the present crash might correspond to the twostep model described in the introduction. Namely, magnetic reconnection proceeds gradually in the first phase in the manner suggested by Kadomtsev. In the second phase, rapid collapsing takes place by some turbulent processes induced by the developed helical hot structure.

Note that the local SX emissivity is a function of the electron temperature, density, and also impurity density. In the present case, for example, the decrement of core SX intensity upon crashing is $20 \%$, while that of ECE is $10 \%$, suggesting that the core density is somewhat decreased upon crashing. The hot structures inferred from SX 


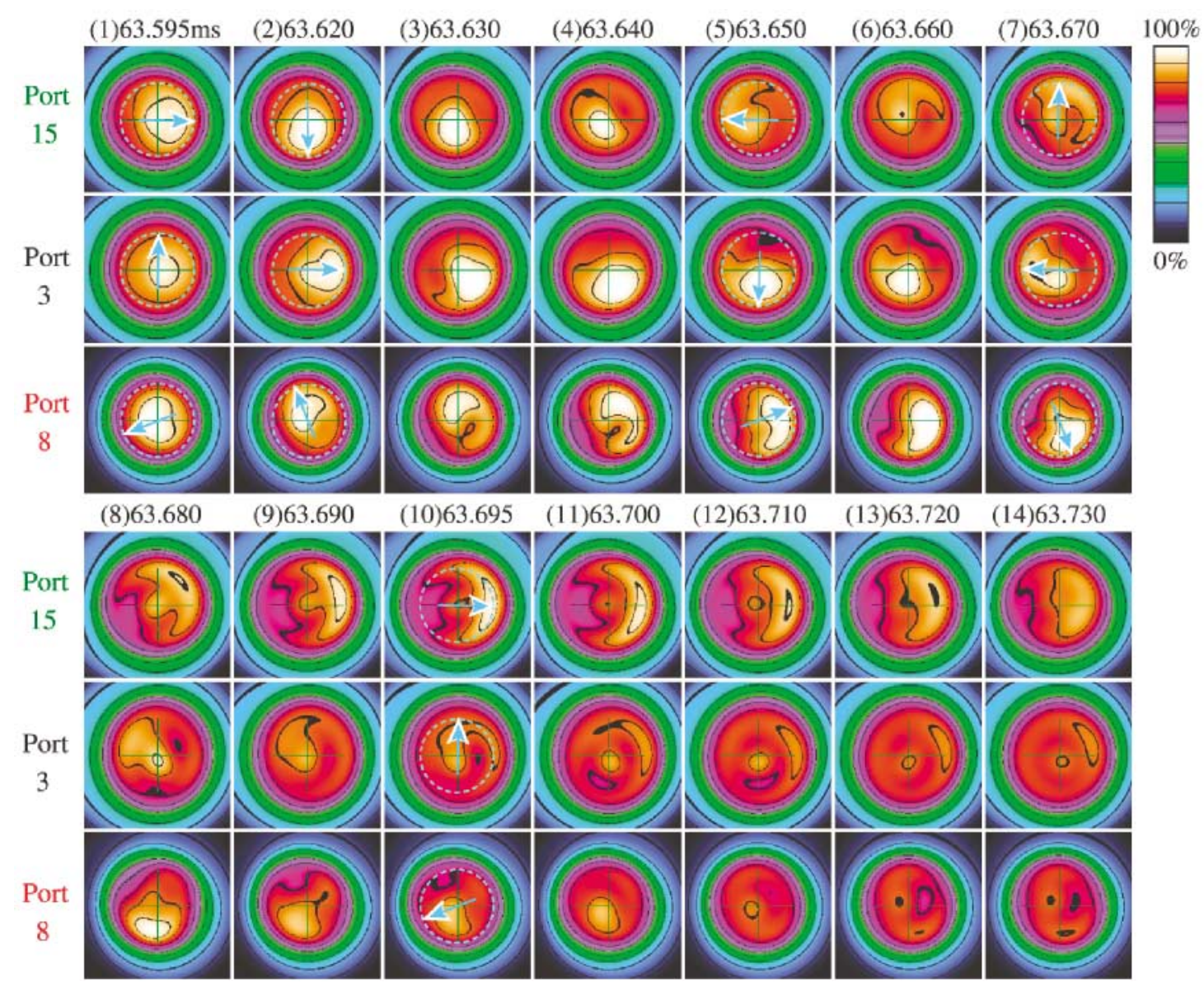

FIG. 2 (color). Time evolutions of the SX images at three ports. In each SX image, the left side is the high field side. The dashed circles denote the inversion circle with $r_{\text {inv }}=63 \mathrm{~mm}$.

contours, however, are also confirmed to be hot by the ECE signals (Fig. 4), although the other contributions cannot be excluded. Especially, the hot crescent at the low field side at the end of the crash is also detected on the ECE signals in the crashes in which the hot crescent happens to come by the ECE port as shown in Fig. 5.
It is remarkable that the hot crescent survives a while at the end of crash with no clear counterpart at the high field side, since the electron temperature should be essentially equal along the field line around the torus. Although this is puzzling and not well understood from the present information on the SX images and ECE signals only, a
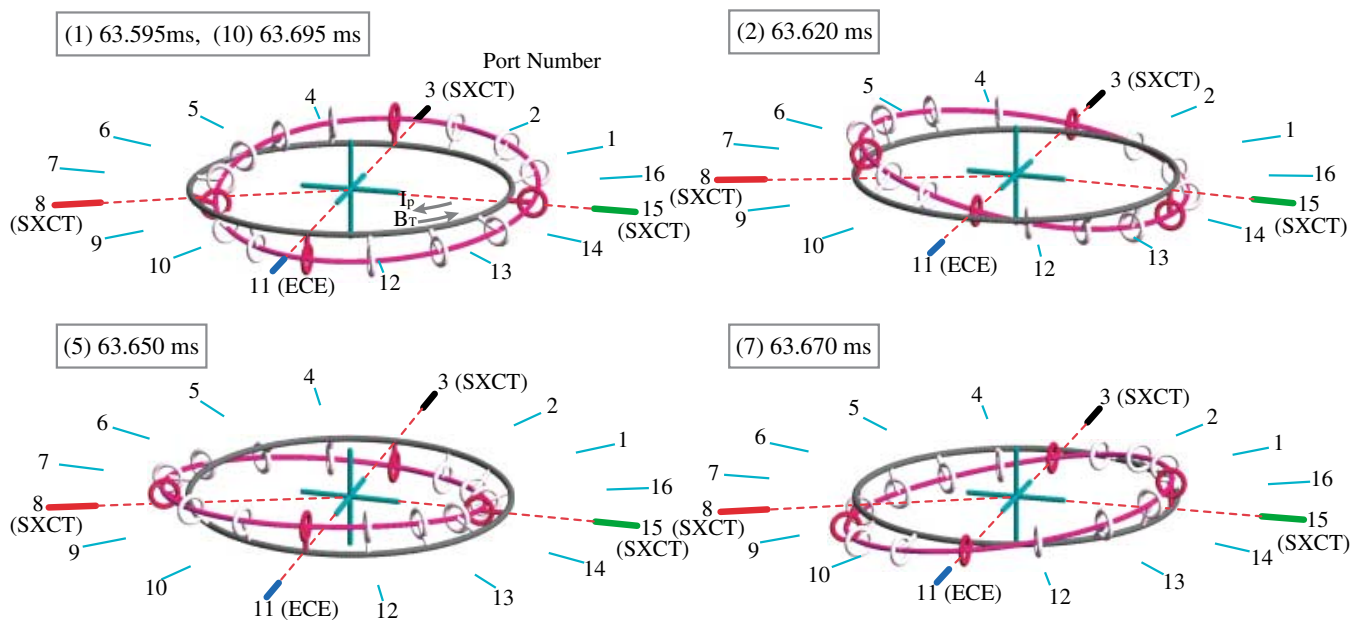

FIG. 3 (color). Schematic presentation of an $m=1 / n=1$ helical hot structure around the torus at the various times in Fig. 2 . The helical structure toroidally rotates in the reverse direction of the plasma current as time proceeds. 


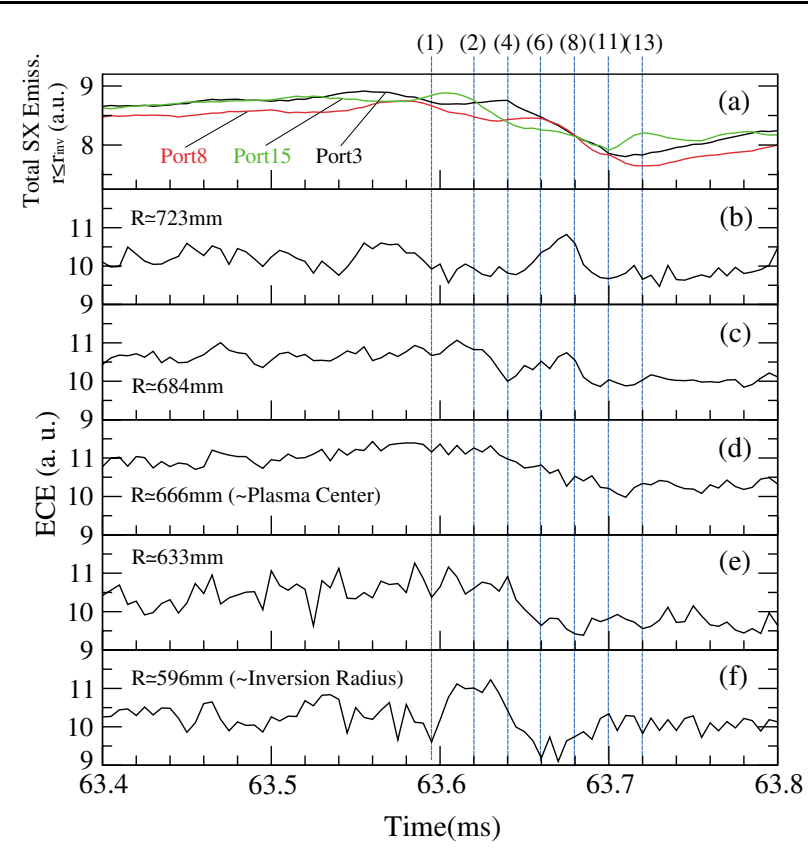

FIG. 4 (color). Time evolutions of (a) total SX emissions inside the inversion radius and (b)-(f) the ECE signals emitted at various radial locations on the midplane at the 11th port section.

possible process is speculated as follows. First, note that space resolution of the present SXCT is not good enough to detect fine hot structures such as the fine hot filaments observed in the Rijnhuizen tokamak project [15]. If many fine hot structures are broadly distributed on a relatively cold area in the plasma cross section, the area would look to be a broad warm region on the SX image. Thus, there might arise a large number of hot fine structures ramified from the crescent structure at the low field side and stretched to the high field side around the torus as a result of strong deformation of the magnetic structure during the second phase of collapsing, which would allow fast thermal diffusion during the crash.

The present type of crashes occupies about $70 \%$ of the total crashes. The next frequent type accompanies an $m=$ $2 / n=2$ mode during the first slow collapsing phase, but the second fast phase looks similar to that of the above normal type without the $m=2$ mode. In addition to these types, there appears a compound sawtooth with $1 \%$ occurrence. This is a sequential event of the partial crash with an $m=2 / n=2$ mode followed by the large crash with an $m=1 / n=1$ mode. In the large crash a hot crescent structure also appears at the low field side at the end of the crash, while the hot core at the high field side is deformed into a broad warm structure spreading from the center to the inversion radius. Such a variety of crashes have been also observed in low $q_{a}(\leq 3)$ Ohmic discharges in Tokamak de Varennes [16] by SXCT and in the Tokamak Fusion Test Reactor [17] by ECE. Both the inversion radius $\left(r_{\text {inv }} / a \simeq 1 / 3\right)$ and the sawtooth ampli-

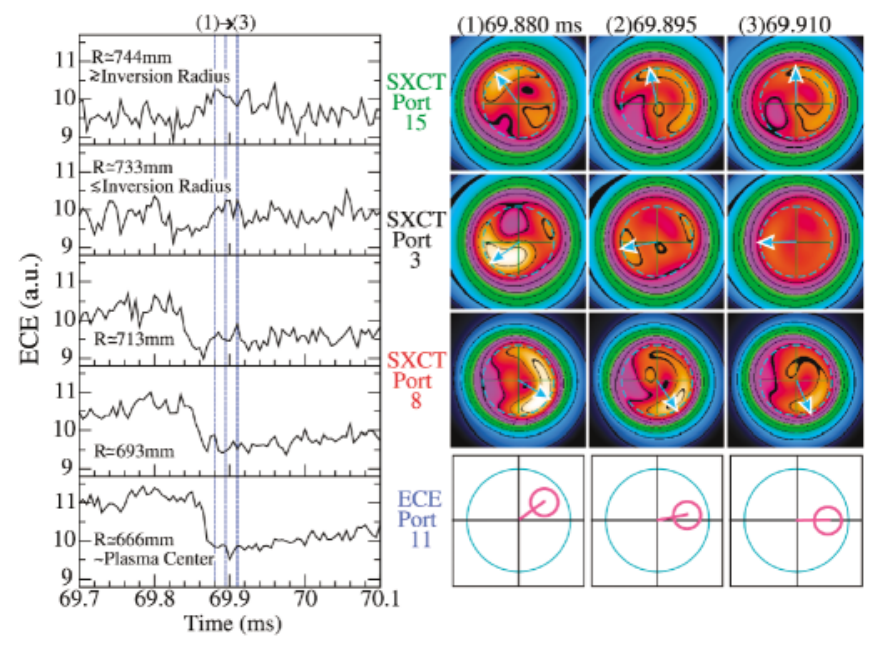

FIG. 5 (color). ECE signals and SXCT images for a crash where the hot crescent happens to come by the ECE port.

tudes $(\Delta A / \mathrm{A} \simeq 15 \%$ and $30 \%$ in Tokamak de Varennes and $\Delta A / A \simeq 20 \%$ and $33 \%$ in WT-3 for the normal and large crashes, respectively, where $\Delta A$ is the decrement of the core SX intensity upon crashes) are large in these low $q_{a}$ discharges.

In summary, a remarkable difference between the hot cores at the low and high field sides during the sawtooth crash has been revealed for the first time by using the MTP SXCT system. In the previous observations by the SXCT at one port, crash processes would look differently depending on the accidental positions of the plasma cross section upon shooting of SX images during the toroidal rotation of plasma, and it would be quite difficult to obtain the whole picture of collapsing all around the torus.

[1] S. von Goeler et al., Phys. Rev. Lett. 33, 1201 (1974).

[2] F. Porcelli, D. Boucher, and M. N. Rosenbluth, Plasma Phys. Controlled Fusion 38, 2163 (1996).

[3] R. J. Hastie, Astrophys. Space Sci. 256, 177 (1998).

[4] B. B. Kadomtsev, Sov. J. Plasma Phys. 1, 389 (1976).

[5] H. Soltwisch, Rev. Sci. Instrum. 57, 1939 (1986).

[6] J. Blum et al., Nucl. Fusion 30, 1475 (1990).

[7] M. Yamada et al., Phys. Plasmas 1, 3269 (1994).

[8] D. Wroblewski and R. T. Snider, Phys. Rev. Lett. 71, 859 (1993).

[9] M. N. Bussac and R. Pellat, Phys. Rev. Lett. 59, 2650 (1987).

[10] A. J. Lichtenberg et al., Nucl. Fusion 32, 495 (1992).

[11] Y. Nagayama et al., Phys. Plasmas 3, 1647 (1996).

[12] S. Yoshimura et al., Phys. Plasmas 7, 276 (2000).

[13] S. Yoshimura et al., Phys. Plasmas 9, 3378 (2002).

[14] Y. Nagayama, J. Appl. Phys. 62, 2702 (1987).

[15] M. N. A. Beurskens et al., Plasma Phys. Controlled Fusion 43, 13 (2001).

[16] C. Janicki et al., Nucl. Fusion 30, 950 (1990).

[17] Y. Nagayama et al., Nucl. Fusion 36, 521 (1996). 